\title{
COMMENTARY
}

TREATMENT OF THE DISLOCATED LENS

If the lens dislocates anteriorly and can be repositioned behind the pupil, a peripheral iridectom $\overrightarrow{\vec{A}_{\mathrm{N}}}$ usually prevents further anterior dislocation of the lens. If the lens is dislocated posteriorly, if should be left alone unless there is some very strong indication for its removal; e.g. when the lent starts to leak and produce a phacolytic glaucoma.

In anterior dislocations of the lens, aspiration of the lens is a reasonable technique especially i there is a strong possibility of vitreous loss at the time of operation.

GAUSE OF GLAUCOMA IN DISLOCATED LENSES

In the Marchesani syndrome changes in the angle are explicable on the basis of a pupillary block $\vec{\omega}$ causing angle closure. Posterior dislocations do not seem to occur in the Marchesani syndromeo There is no evidence that a posteriorly dislocated lens causes glaucoma through stimulation of tho. ciliary body.

GLAUCOMATOUS CHANGES IN HOMOGYSTINURIA

A great many patients with homocystinuria who develop optic atrophy with cupping resembling aิ glaucomatous optic atıophy in the absence of a raised intraocular pressure, also have increasedf platelet stickiness. Dr. Spaeth felt that the platelet stickiness changes were universal in homocystinuria and that if they are not demonstrated this is probably the fault of the technique. As there is a direct relationship between the amount of stickiness and the level of the homocystine iñ seems possible that the increased platelet stickiness produces an occlusive vascular disease and henceatrophy of the optic nerve head. Homocystinuria is an extremely rare disease even amongst the mentally retarded. Dr. Spaeth surveyed I0,00o individuals who were mentally retarded and only found three cases of homocystinuria. However, as it now appears that some of these patients are likely to be treatable with low methionine diets and/or pyridoxine, it seems worth while locating them early. Very little is yet known of the -natural history of the condition. One young girl followed by Dr. Spaeth since 1965 and treated with diet and pyridoxine has so far had no ocular complications命 The most satisfactory screening test for homocystinuria is the silver modification of the cyanide $\underset{\bar{F}}{\overrightarrow{1}}$ nitroprusside test (Winston and Barber), which practically eliminates most of the false positiveş found with the original cyanide-nitroprusside test.

\section{Treatment of juvenile glaucoma}

\section{J. HETHERINGTON}

San Francisco, U.S.A.

\section{GOMMENTARY}

Patients with juvenile glaucoma respond extremely badly to the standard surgical procedures, bu many have anterior insertions of the iris and angles which look rather like those seen in infantilew glaucoma. Some of these patients do well with goniotomy using the Lister modification of the Barkan knife made by Grieshaber. This is a sharp instrument making it much easier to enter th\& anterior chamber and to dissect over the anterior aspect of the trabecular meshwork. Bleeding which cannot be readily washed out as with infants, is particularly severe in young patients, but has never been so disastrous as to require further surgery. If goniotomy has been unsuccessful, Professor Richardson has found that goniopuncture is, occasionally, a worthwhile procedure, though it may not succeed in more than one in ten or one in fifteen.

In young children with the Sturge-Weber syndrome it was necessary to use cyclocryo-therapy as a primary procedure. This could be repeated on several occasions if necessary. 